\title{
Development and characterization of TrMab-6, a novel anti-TROP2 monoclonal antibody for antigen detection in breast cancer
}

\author{
YUSUKE SAYAMA $^{1}$, MIKA K. KANEKO ${ }^{1}$ and YUKINARI KATO ${ }^{1,2}$ \\ ${ }^{1}$ Department of Antibody Drug Development, Tohoku University Graduate School of Medicine; \\ ${ }^{2}$ New Industry Creation Hatchery Center, Tohoku University, Sendai, Miyagi 980-8575, Japan
}

Received June 26, 2020; Accepted September 15, 2020

DOI: $10.3892 / \mathrm{mmr} .2020 .11731$

\begin{abstract}
Trophoblast cell-surface antigen 2 (TROP2) is a type I transmembrane glycoprotein that is overexpressed in a number of cancer types, including triple-negative breast cancer. The current study aimed to develop a highly sensitive and specific monoclonal antibody (mAb) targeting TROP2, which could be used to evaluate TROP2 expression using flow cytometry, western blot analysis and immunohistochemistry by employing the Cell-Based Immunization and Screening (CBIS) method. The established anti-TROP2 mAb, TrMab-6 (mouse $\operatorname{IgG}_{2 \mathrm{~b}}, \kappa$ ), detected TROP2 on PA-tagged TROP2-overexpressing Chinese hamster ovary-K1 (CHO/TROP2-PA) and breast cancer cell lines, including MCF7 and BT-474 using flow cytometry. Western blot analysis indicated a $40 \mathrm{kDa}$ band in lysates prepared from CHO/TROP2-PA, MCF7 and BT-474 cells. Furthermore, TROP2 in 57/61 (93.4\%) of the breast cancer specimens was strongly detected using immunohistochemical analysis with TrMab-6. In conclusion, the current study demonstrated that TrMab- 6 may be a valuable tool for the detection of TROP 2 in a wide variety of breast cancer types.
\end{abstract}

Correspondence to: Professor Yukinari Kato, New Industry Creation Hatchery Center, Tohoku University, 2-1 Seiryo-machi, Aoba-ku, Sendai, Miyagi 980-8575, Japan

E-mail: yukinarikato@med.tohoku.ac.jp

Abbreviations: ADCC, antibody-dependent cellular cytotoxicity; CBIS, Cell-Based Immunization and Screening; CHO, Chinese hamster ovary; DMEM, Dulbecco's modified Eagle's medium; EDTA, ethylenediaminetetraacetic acid; $\mathrm{mAb}$, monoclonal antibody; H\&E, hematoxylin and eosin; RPMI, Roswell Park Memorial Institute; TACTD2, tumor-associated calcium signal transducer 2; TROP2, trophoblast cell-surface antigen 2

Key words: TROP2, monoclonal antibody, CBIS, breast cancer

\section{Introduction}

Breast cancer is by far the most common malignant tumor in women. In 2018, there were 2,088,849 new breast cases $(11.6 \%$ of the total cancer cases) and 626,679 deaths $(6.6 \%$ of the total cancer deaths) worldwide (1). Surgery, radiotherapy, chemotherapy, and molecular targeted therapies are currently used for breast cancer treatment; however, effective therapies for patients diagnosed with triple-negative breast cancer [TNBC; i.e., those that are negative for estrogen receptor (ER), progesterone receptor (PR), and human epidermal growth factor receptor 2 (HER2)] remain limited (2-4). TNBC accounts for $\sim 15 \%$ of invasive breast cancers; moreover, it tends to be aggressive and is associated with a poor prognosis $(2,5,6)$. TNBC is more common in young women than in older women and is frequently associated with invasion and metastatic disease (2,5-7). As such, highly sensitive and specific monoclonal antibodies (mAbs) are required to facilitate the diagnosis of and treatment decisions for this breast cancer subtype.

The trophoblast cell-surface antigen (TROP2), also known as human tumor-associated calcium signal transducer (TACSTD2), is a type I transmembrane glycoprotein originally identified in human trophoblast cells (8-10). Previously, Schon and Orfanos reported that tunicamycin treatment of living cells and $\mathrm{N}$-glycanase digestion of immunopurified TROP2 revealed that the molecular heterogeneity of TROP2 is due to the different $N$-glycosylation in normal and transformed keratinocytes (11). In transformed keratinocytes, two distinct precursor proteins at 38 and $42 \mathrm{kDa}$ were detected, whereas in normal cells the $38-\mathrm{kDa}$ signal was dramatically decreased, indicating that quantitative and qualitative changes of $N$-glycan of TROP2 are associated with the transformation process of human keratinocytes. TROP2 is highly expressed in several cancers and may play a critical role in tumor progression in association with the pathways involving both the extracellular signal-related kinase (ERK) and c-Jun N-terminal kinase (JNK) $(12,13)$. The expression of TROP2 has been reported in more than $85 \%$ of all tumors; as such, TROP2 may be a useful marker for cancer diagnosis and immunotherapy $(2,14,15)$. It has also been identified in the stem cells of various tissues, including basal cells, all of which are capable of self-renewal, regeneration, and differentiation $(2,16,17)$. Several mAbs 
targeting TROP2 are currently evaluated in clinical trials, including PF-06664178 (12,18), IMMU-132 $(12,19,20)$, and DS-1062a $(12,21)$.

In our previous studies, we developed the Cell-Based Immunization and Screening (CBIS) method; in this method, cell lines are used exclusively for both immunization and screening (22). CBIS has been employed to develop sensitive and specific $\mathrm{mAbs}$ against numerous transmembrane proteins, including CD19 (23), CD20 (24), CD44 (25), CD133 (22), and PD-L1 (26). Of note, mAbs developed using this method have proven to be extremely useful in flow cytometry, Western blot, and immunohistochemical analyses.

In this study, we developed novel anti-TROP2 mAbs and evaluated their capacity to target breast cancer cells using flow cytometry, Western blot, and immunohistochemical analyses.

\section{Materials and methods}

Plasmid preparation. Human TROP2 DNA was synthesized commerciallybyThermoFisherScientific(Waltham,MA,USA). TROP2 DNA with an N-terminal PA16 tag (27) and a C-terminal RAP tag (28)/MAP tag (29) (PA16-TROP2-RAP-MAP) was subcloned into the pCAG-Ble expression vector (FUJIFILM Wako Pure Chemical Corporation) using an In-Fusion HD Cloning Kit (Takara Bio, Inc.); the recombinant expression vector was named pCAG/PA16-TROP2-RAP-MAP. TROP2 DNA with a C-terminal PA tag (27) alone was also subcloned into the pCAG-Ble vector using an In-Fusion HD Cloning $\mathrm{Kit}$; this expression vector was named pCAG/TROP2-PA. The amino acid sequences of each tag are as follows: PA16 tag, 16 amino acids (GLEGGVAMPGAEDDVV); PA tag, 12 amino acids (GVAMPGAEDDVV); RAP tag, 12 amino acids (DMVNPGLEDRIE); and MAP tag, 12 amino acids (GDGMVPPGIEDK).

Cell lines. Chinese hamster ovary (CHO)-K1, P3X63Ag8U.1 (P3U1), BT-474, Lec1, Lec2, and Lec8 cell lines were obtained from the American Type Culture Collection (ATCC; Manassas, VA, USA). MCF7 was obtained from the Cell Resource Center for Biomedical Research, Institute of Development, Aging and Cancer, Tohoku University (Miyagi, Japan).

CHO-K1 cells that overexpress TROP2-PA (CHO/TROP2-PA) and PA16-TROP2-RAP-MAP (CHO/PA16-TROP2-RAP-MAP) were generated by transfection of pCAG/TROP2-PA and pCAG/PA16-TROP2-RAP-MAP to $\mathrm{CHO}-\mathrm{K} 1$ cells, respectively, using Lipofectamine LTX Reagent (Thermo Fisher Scientific, Inc.). Cell lines Lec1/TROP2, Lec2/TROP2, and Lec8/TROP2 were generated by transfection of pCAG/TROP2-PA to Lec1, Lec2, and Lec8 cells, respectively, using the Neon Transfection System (Thermo Fisher Scientific, Inc.). Several days after the transfection, the transfected cells were confirmed as TROP2-positive by flow cytometry (EC800, Sony Corp.) using a commercial anti-TROP2 antibody (Cat\#LS-C489657, LS Bio). The transfected cells were selected by limiting dilution culture and cultivation in the medium containing $0.5 \mathrm{mg} / \mathrm{ml}$ of zeocin (InvivoGen). We confirmed the transfection efficiency using western blotting.

The TROP2 gene-deleted cell line, MCF7/TROP2-KO (BINDS-29), was generated by transfection of CRISPR/Cas9 plasmids targeting TROP2 using the Neon Transfection System (Thermo Fisher Scientific, Inc.). Stable transfectants were established by cell sorting using SH800 (Sony Corp.).

CHO-K1,CHO/PA16-TROP2-RAP-MAP,CHO/TROP2-PA, P3U1, MCF7, Lec1/TROP2, Lec2/TROP2, Lec8/TROP2, and BINDS-29 cells were cultured in Roswell Park Memorial Institute (RPMI)-1640 medium (Nacalai Tesque, Inc., Kyoto, Japan); BT-474 cells were cultured in Dulbecco's modified Eagle's medium (DMEM; Nacalai Tesque, Inc.). All media were supplemented with $10 \%$ heat-inactivated fetal bovine serum (FBS; Thermo Fisher Scientific, Inc.), $100 \mathrm{U} / \mathrm{ml}$ penicillin, $100 \mu \mathrm{g} / \mathrm{ml}$ streptomycin, and $0.25 \mu \mathrm{g} / \mathrm{ml}$ amphotericin $\mathrm{B}$ (Nacalai Tesque, Inc.). Cells were grown in an incubator at $37^{\circ} \mathrm{C}$ with humidity and $5 \% \mathrm{CO}_{2}$ and $95 \%$ air atmosphere.

Animals. Female BALB/c mice (6 weeks old) were purchased from CLEA Japan and kept under specific pathogen-free conditions. All animal experiments were conducted in accordance with the relevant guidelines and regulations in order to minimize animal suffering and distress in the laboratory. The Animal Care and Use Committee of Tohoku University approved all the animal experiments (permit no. 2019NiA-001). Mice were euthanized by cervical dislocation under inhalation anesthesia using $2 \%$ of isoflurane, and the death was verified to be respiratory and cardiac arrest.

Hybridoma production. We employed CBIS to develop new $\mathrm{mAbs}$ against TROP2. Two mice were immunized with CHO/PA16-TROP2-RAP-MAP cells $\left(1 \times 10^{8}\right)$ via the intraperitoneal route (i.p.) together with the Imject Alum (Thermo Fisher Scientific, Inc.). After several additional immunizations, a booster immunization was administered via the i.p. route 2 days before spleen cell collection. Mice were euthanized by cervical dislocation under inhalation anesthesia using isoflurane, and the death was verified to be respiratory and cardiac arrest. We chopped spleens, and collected spleen cells using serum-free RPIM-1640 medium. We further broke the red blood cells with $3 \mathrm{ml}$ of Red Blood Cell Lysing Buffer Hybri-Max (Sigma-Aldrich Corp.) at $37^{\circ} \mathrm{C}$ for $1 \mathrm{~min}$, and washed the spleen cells using serum-free RPIM-1640 medium. The collected spleen cells were fused with P3U1 mouse myeloma cells using polyethylene glycol 1500 (Roche Diagnostics) (30,31); the resulting hybridomas were selected in RPMI medium, including hypoxanthine, aminopterin, and thymidine (Thermo Fisher Scientific, Inc.). The culture supernatants were screened via flow cytometry using $\mathrm{CHO} / \mathrm{TROP} 2-\mathrm{PA}$ and $\mathrm{CHO}-\mathrm{K} 1$ cells.

Flow cytometry. Cells were collected following a brief exposure to $0.25 \%$ trypsin and $1 \mathrm{mM}$ ethylenediaminetetraacetic acid (EDTA; Nacalai Tesque, Inc.). The cells were washed with $0.1 \%$ bovine serum albumin in phosphate-buffered saline (PBS) and treated with anti-TROP2 mAbs, such as TrMab-6 (1 $\mu \mathrm{g} / \mathrm{ml})$ or EPR20043 (1/60 dilution; Abcam) for $30 \mathrm{~min}$ at $4{ }^{\circ} \mathrm{C}$. After incubation, the cells were treated with Alexa Fluor 488-conjugated anti-mouse IgG (1:1,000; Cell Signaling Technology, Inc.) or Alexa Fluor 488-conjugated anti-rabbit IgG (1:1,000; Cell Signaling Technology, Inc.). Fluorescence data were collected using SA3800 Spectral Cell Analyzer (Sony Corp.) and analyzed using FlowJo (BD Biosciences). 
Determination of the binding affinity. MCF7 or BT-474 cells $\left(2 \times 10^{5}\right)$ were suspended in $100 \mu \mathrm{g}$ of serially diluted TrMab-6 $(6 \mathrm{ng} / \mathrm{ml}-100 \mu \mathrm{g} / \mathrm{ml})$ for $30 \mathrm{~min}$ at $4^{\circ} \mathrm{C}$, followed by the addition of Alexa Flour 488-conjugated anti-mouse IgG (1:200; Cell Signaling Technologies, Inc.). Fluorescence data were collected using a cell analyzer (EC800). The dissociation constant $\left(K_{\mathrm{D}}\right)$ was calculated by fitting the binding isotherms to built-in, one-site binding models in GraphPad Prism 8 (GraphPad Software, Inc.).

Western blot analysis. Cell lysates $(10 \mu \mathrm{g})$ were boiled in sodium dodecyl sulfate (SDS) sample buffer (Nacalai Tesque, Inc.). Proteins were separated on 5-20\% polyacrylamide gels (FUJIFILM Wako Pure Chemical Corporation) and transferred onto polyvinylidene difluoride (PVDF) membranes (Merck KGaA). After blocking with $4 \%$ skim milk (Nacalai Tesque, Inc.) in PBS with $0.05 \%$ Tween-20, the membranes were incubated with 1 or $5 \mu \mathrm{g} / \mathrm{ml}$ of TrMab-6, 1/2000 dilution of EPR20043 (Abcam), $1 \mu \mathrm{g} / \mathrm{ml}$ of NZ-1 (anti-PA tag), or $1 \mu \mathrm{g} / \mathrm{ml}$ of anti- $\beta$-actin (clone AC-15; Sigma-Aldrich Corp.). This was followed by incubation with peroxidase-conjugated anti-mouse immunoglobulins (Agilent Technologies Inc.; diluted 1:1,000) to detect TrMab- 6 and anti- $\beta$-actin, peroxidase-conjugated anti-rabbit immunoglobulins (Agilent Technologies Inc.; diluted 1:1,000) to detect EPR20043, or anti-rat IgG (Sigma-Aldrich Corp; diluted 1:10,000) to detect NZ-1, respectively. Finally, protein bands were detected with ImmunoStar LD (FUJIFILM Wako Pure Chemical Corporation) using a Sayaca-Imager (DRC Co. Ltd.).

Immunohistochemical analysis. Paraffin-embedded tissue sections of the breast cancer tissue array (Cat\#T8235721-5, Lot\#B104066; BioChain, San Francisco, CA, USA) were autoclaved in EnVision FLEX Target Retrieval Solution High pH (Agilent Technologies, Inc.) for 20 min. After blocking with SuperBlock T20 (Thermo Fisher Scientific, Inc.), tissue sections were incubated with TrMab-6 $(5 \mu \mathrm{g} / \mathrm{ml})$ or EPR20043 (1/500 dilution; Abcam) for $1 \mathrm{~h}$ at room temperature and then treated with the EnVision+ Kit for mouse (Agilent Technologies Inc.) and EnVision+ Kit for rabbit (Agilent Technologies Inc.) for $30 \mathrm{~min}$, respectively. Color was developed using 3,3'-diaminobenzidine tetrahydrochloride (DAB; Agilent Technologies Inc.) for $2 \mathrm{~min}$. Counterstaining was performed with hematoxylin (FUJIFILM Wako Pure Chemical Corporation).

\section{Results}

Development of novel anti-TROP2 mAbs using the CBIS method. We immunized two mice with CHO/PA16-TROP2RAP-MAP cells and anti-TROP2 mAbs were screened via flow cytometry (Fig. 1). The first screening approach identified strong signals from $\mathrm{CHO} / \mathrm{TROP} 2-\mathrm{PA}$ cells and weak to no signals from $\mathrm{CHO}-\mathrm{K} 1$ cells using hybridoma supernatants from 90 of the 956 wells $(9.4 \%)$. The second screening approach identified strong signals from MCF7 cells from 84 of the 90 hybridoma supernatants identified in the earlier step (93.3\%). After limiting dilution, we established 30 positive clones. Further screening via Western blot and immunohistochemistry led to the establishment of TrMab-6. The subclass
1. Immunization of cell lines

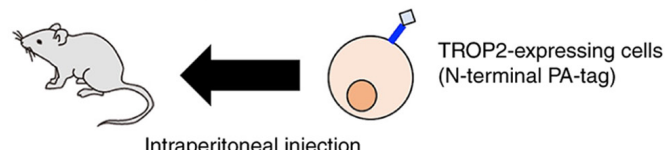

2. Production of hybridomas

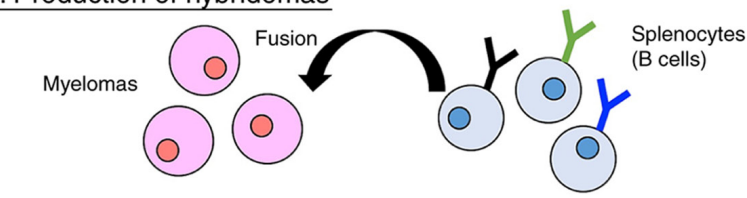

3. Screening (flow cytometry)

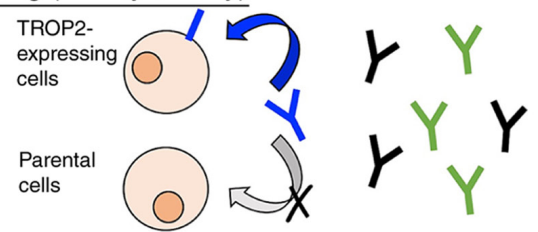

4. Cloning of hybridomas

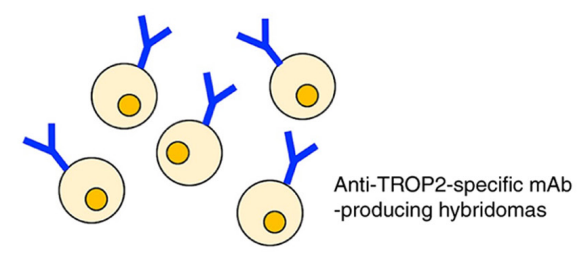

Figure 1. Illustration of Cell-Based Immunization and Screening. A total of two mice were immunized with CHO/PA16-TROP2-RAP-MAP cells, and anti-TROP2 monoclonal antibodies were screened using flow cytometry. TROP2, TROP2, trophoblast cell-surface antigen 2.

of TrMab-6 was determined to be mouse $\operatorname{IgG}_{2 \mathrm{~b}}$ as shown in Fig. S1A.

Flow cytometry analysis. We developed several transfectants, such as CHO/TROP2-PA, Lec1/TROP2, Lec2/TROP2, and Lec8/TROP2, and the transfection efficiency was confirmed using an anti-PA tag $\mathrm{mAb}$ (NZ-1) by Western blot analysis (Fig.S1B). Then, we performed flow cytometry targeting several relevant cell lines in order to characterize antigen detection using TrMab-6 (Fig. 2). TrMab-6 detected CHO/TROP2-PA cells, but not parental CHO-K1 cells. TrMab-6 also detected endogenous TROP2 on human breast cancer cell lines, including MCF7 and BT-474. Contrarily, TrMab-6 did not react with BINDS-29 (TROP2-gene-deleted MCF7 cells). Taken together, these results suggested that TrMab- 6 is specific for TROP2. As shown in Fig. S2, another anti-TROP2 mAb (clone EPR20043) weakly reacted with MCF7, but did not react with BT-474 although TrMab-6 strongly reacted with both MCF7 and BT-474, indicating that TrMab-6 is more useful for flow cytometry than EPR20043 although EPR20043 was shown to be useful in all applications, such as flow cytometry, Western blot, and immunohistochemical analyses (Table SI).

Next, we investigated whether the epitope of TrMab-6 is associated with glycans. Thus, we performed flow cytometry using TROP2-transfected glycan-deficient CHO cells, including those deficient in Lec1 ( $N$-glycan-deficient), Lec2 (sialic acid-deficient), and Lec8 (galactose-deficient) cells. As presented in Fig. 2, TrMab- 6 reacted with Lec1/TROP2,Lec2/TROP2, and Lec8/TROP2 cells to an extent 
A
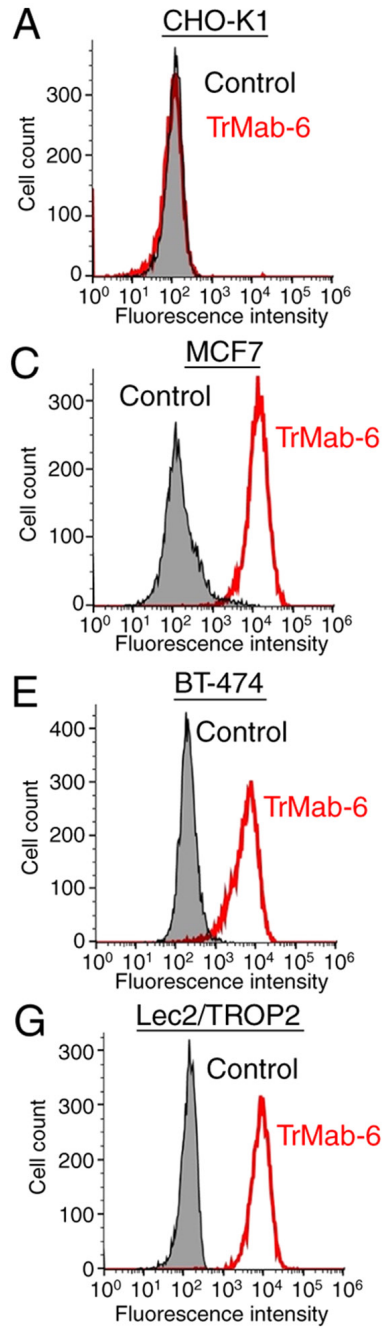
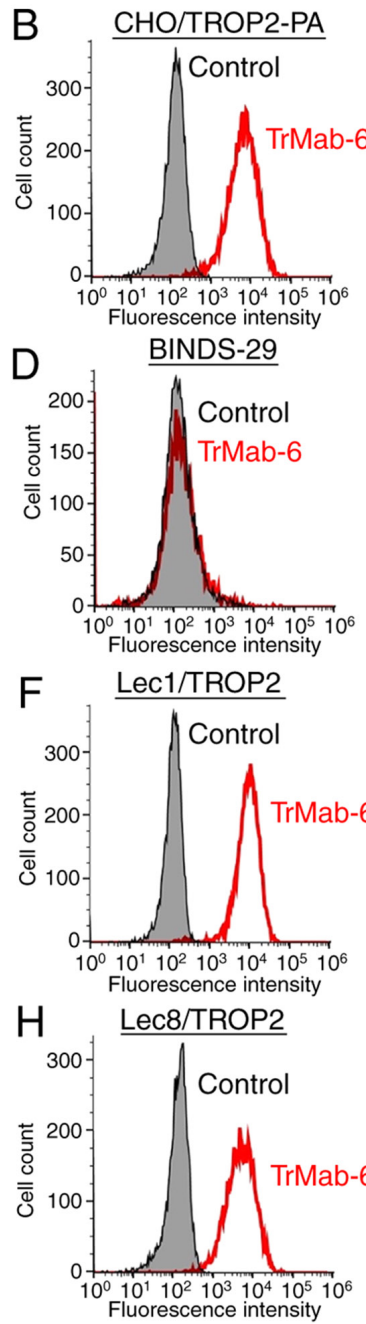

Figure 2. Flow cytometric detection of TROP2 using TrMab-6. (A) CHO-K1, (B) CHO/TROP2-PA, (C) MCF7, (D) BINDS-29 (MCF7/TROP2-KO), (E) BT-474, (F) Lec1/TROP2, (G) Lec2/TROP2 and (H) Lec8/TROP2 cells were incubated with TrMab-6 $(1 \mu \mathrm{g} / \mathrm{ml}$; red line) or $0.1 \%$ BSA in PBS (grey) for $30 \mathrm{~min}$, followed by Alexa Fluor 488-conjugated secondary antibodies. Fluorescence data were collected using a cell analyzer. TROP2, trophoblast cell-surface antigen 2 .

indistinguishable from that observed with $\mathrm{CHO} / \mathrm{TROP} 2-\mathrm{PA}$. These results indicated that the binding epitope recognized by TrMab-6 was unlikely to be associated with glycans.

Determination of the binding affinity using TrMab-6 against breast cancers by flow cytometry. To determine the binding affinity of TrMab-6, we conducted kinetic analysis of the interaction of TrMab- 6 with MCF7 and BT-474 cells via flow cytometry. The $K_{\mathrm{D}}$ of TrMab- 6 was determined to be $6.5 \times 10^{-9} \mathrm{M}$ when targeting MCF7 cells and $1.1 \times 10^{-10} \mathrm{M}$ for BT-474 cells (Fig. 3). These results indicated that TrMab-6 binds with high affinity to TROP2-expressing breast cancer cells.

Western blot analyses. TrMab-6 binding identified TROP2 as an immunoreactive band with an estimated $40 \mathrm{kDa}$ band in lysates prepared from $\mathrm{CHO} / \mathrm{TROP} 2-\mathrm{PA}, \mathrm{MCF} 7$, and BT-474 cells; no immunoreactive bands were found in $\mathrm{CHO}-\mathrm{K} 1$ and TROP2-gene-deleted MCF7 (BINDS-29) cells (Fig. 4), again confirming its specificity for TROP2. TrMab-6 also detected

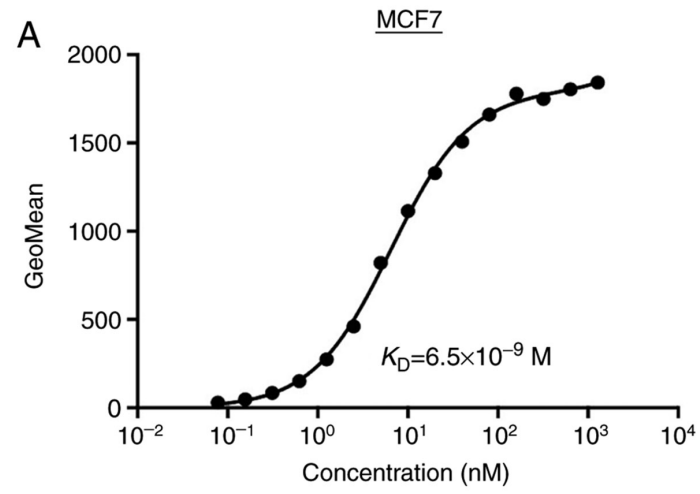

B $\quad \underline{\text { BT- } 474}$

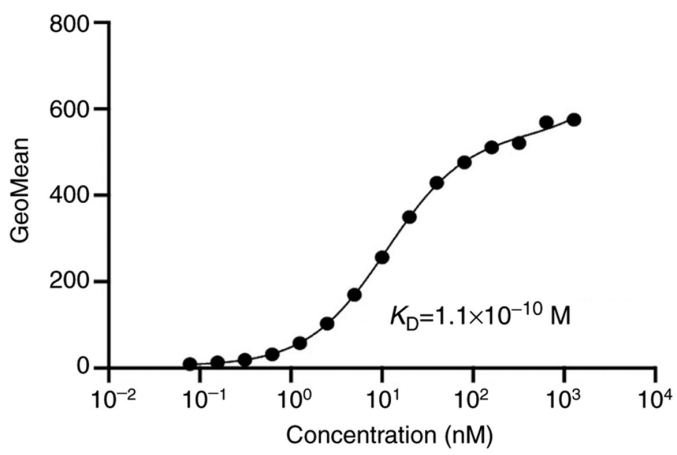

Figure 3. Flow cytometric determination of the binding affinity of TrMab-6 for breast cancer cells. (A) MCF7 or (B) BT-474 cells were suspended in $100 \mathrm{ul}$ of serially diluted antibodies $(6 \mathrm{ng} / \mathrm{ml}$ to $100 \mu \mathrm{g} / \mathrm{ml})$, followed by incubation with Alexa Fluor 488-conjugated secondary antibodies. Fluorescence data were collected using a cell analyzer; GeoMean, geometric mean of fluorescence intensity.

TROP2 of Lec1/TROP2, Lec2/TROP2, and Lec8/TROP2. Although TROP2 proteins, which were expressed in Lec1/TROP2 and Lec8/TROP2, were detected in lower molecular weight compared with CHO/TROP2-PA and Lec2/TROP2, the intensity by TrMab-6 was similar among those cell lines, indicating that the binding epitope of TrMab-6 is independent of glycans. An anti-PA tag mAb (NZ-1) also detected TROP2 bands in lysates of CHO/TROP2-PA, Lec1/TROP2, Lec2/TROP2, and Lec8/TROP2 cells. These results indicated that TrMab- 6 could be used to detect TROP2 expressed by breast cancer cells via Western blot.

We compared the reactivity of TrMab- 6 and another anti-TROP2 mAb (clone EPR20043) in Western blot analysis. As shown in Fig. S3, both TrMab-6 and EPR20043 strongly detected TROP2 from both MCF7 and BT-474, indicating that both TrMab-6 and EPR20043 are useful for Western blot analysis.

Immunohistochemical analyses against breast cancer. We then used TrMab- 6 to target clinical specimens of human breast cancer tissue via immunohistochemical analysis (Table I). TrMab-6 detected TROP2 in 57/61 of the breast cancer specimens (93.4\%; Table II). Among these specimens were 50/54 cases (93.1\%) of invasive ductal carcinoma (Table II). Typical TrMab-6-associated staining patterns in the specimens of invasive ductal carcinomas are presented in Fig. 5A and B. Hematoxylin and eosin (H\&E) staining of invasive ductal carcinoma tissue is presented in Fig. 5C and D. Furthermore, 
A

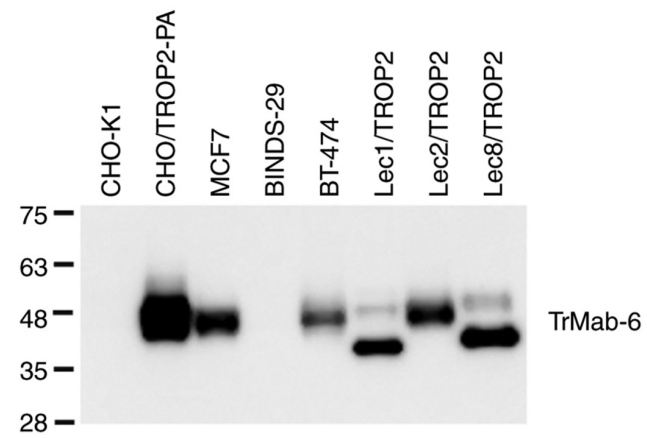

B

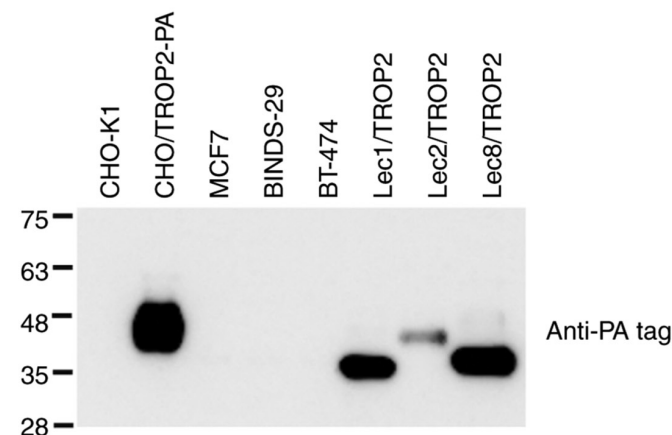

C

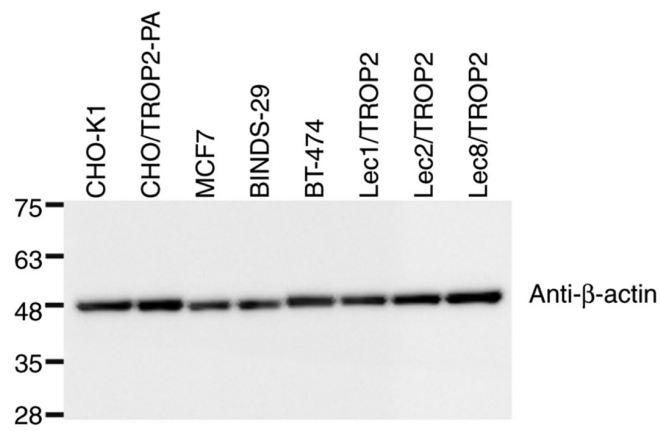

Figure 4. Detection of TROP2 with TrMab-6 using western blot analysis The cell lysates $(10 \mu \mathrm{g})$ of CHO-K1, CHO/TROP2-PA, MCF7, BINDS-29 (MCF7/TROP2-KO), BT-474, Lec1/TROP2, Lec2/TROP2 and Lec8/TROP2 were subjected to SDS-PAGE and transferred onto the PVDF membranes. The membranes were incubated with $1 \mu \mathrm{g} / \mathrm{ml}$ of (A) TrMab-6, an (B) anti-PA $\mathrm{mAb}(\mathrm{NZ}-1)$ and an (C) anti- $\beta$-actin mAb (AC-15), followed by secondary antibodies. TROP2, trophoblast cell-surface antigen 2 .

TrMab- 6 detected TROP2 in 4/4 cases (100\%) of invasive lobular carcinoma, $2 / 2$ cases $(100 \%)$ of adenocarcinoma, and $1 / 1$ case $(100 \%)$ of medullary carcinoma (Table II); the typical staining patterns of invasive lobular carcinoma are presented in Fig. $5 \mathrm{E}$ and $\mathrm{F}$, and $\mathrm{H} \& \mathrm{E}$ staining was performed as presented in Fig. 5G and H. Among the 61 breast cancer cases, 30/61 cases (49.2\%) were stained strongly positive, $18 / 61$ cases $(29.5 \%)$ were stained moderately positive, and 9/61 cases (14.8\%) were stained weakly positive by TrMab-6 (Table II). We obtained the information about ER, PR, and HER2 (Table I). To determine HER 2 expression, we used an anti-HER $2 \mathrm{mAb}$ (clone $\mathrm{H}_{2}$ Mab-77) (32). Among 61 breast cancers, 31 cases (50.8\%) were determined to be triple-negative (Table III). Interestingly, 28/31 (90.3\%) were stained by TrMab-6; especially, 17/31 (54.8\%) were stained strongly positive by TrMab-6 (Table III), indicating that triple-negative breast cancers should be an ideal target of anti-TROP2 mAbs, including TrMab-6.
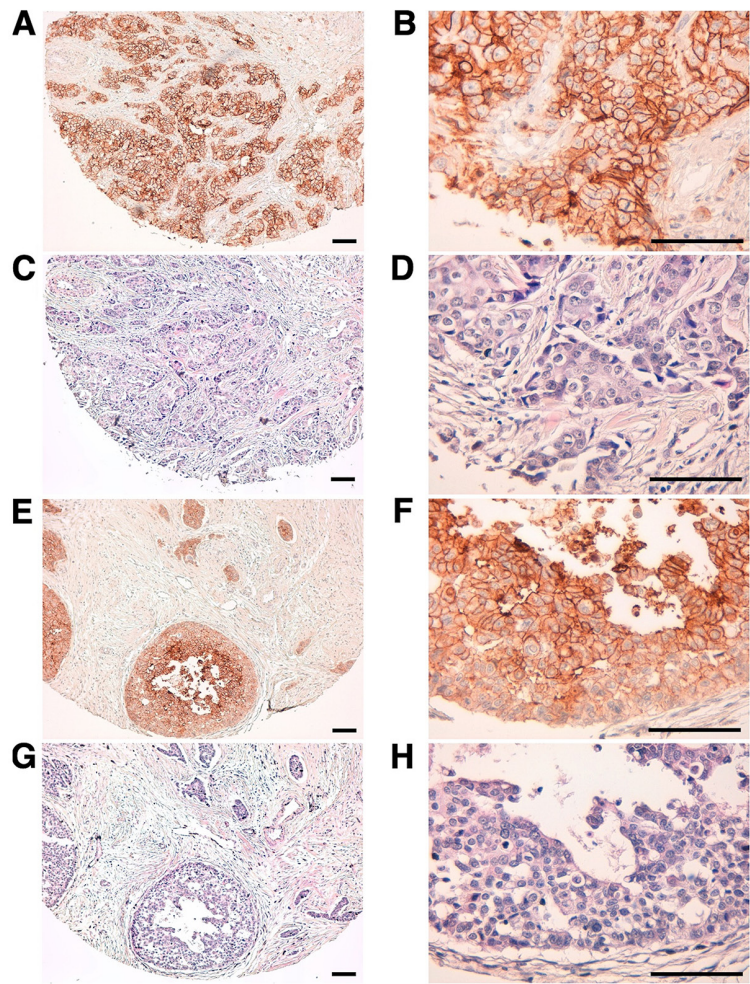

Figure 5. Detection of TROP2 in breast cancer specimens using immunohistochemical analysis with TrMab-6. (A and B) Tissue sections of patients with human breast cancer (invasive ductal carcinoma, Case No. 5) were incubated with $5 \mu \mathrm{g} / \mathrm{ml}$ of TrMab-6 and then treated with the EnVision+ kit. Tissues were counterstained with hematoxylin. (C and D) H\&E staining was performed using consecutive breast cancer tissues (invasive ductal carcinoma, Case No. 14). (E and F) Tissue sections of patients with human breast cancer (invasive lobular carcinoma) were incubated with $5 \mu \mathrm{g} / \mathrm{ml}$ of TrMab-6 and treated with a EnVision+ kit. Counterstaining was performed with hematoxylin. ( $\mathrm{G}$ and $\mathrm{H}$ ) $\mathrm{H} \& \mathrm{E}$ staining was performed using consecutive breast cancer tissues (invasive lobular carcinoma). Scale bar, $100 \mu \mathrm{m}$. TROP2, trophoblast cell-surface antigen 2; H\&E, hematoxylin and eosin.

We compared the reactivity of TrMab-6 and another anti-TROP2 $\mathrm{mAb}$ (clone EPR20043) in immunohistochemical analysis. As shown in Table I, EPR20043 stained 60/61 (98.4\%) breast cancer tissues, although TrMab-6 stained 57/61 (93.4\%) breast cancer tissues, indicating that EPR20043 is more useful for immunohistochemical analysis than TrMab-6.

\section{Discussion}

Generating a $\mathrm{mAb}$ that can be utilized for multiple applications, including flow cytometry, Western blot, and immunohistochemistry, is usually difficult. Using the CBIS method, in which antigen-expressing cell lines are used for both immunization and screening (22), we have developed numerous useful mAbs that target membrane proteins, including CD19 (23), CD20 (24), CD44 (25), CD133 (22), PD-L1 (26), and podoplanin (PDPN) (33-36). Among these unique targets, CD20 has four membrane-spanning domains and only two small extracellular domains that include amino acids 72-80 and 142-182 (37,38). Although there are several commercially available mAbs that interact with amino acids 142-182 of CD20 and are specifically useful in flow cytometry, there are no available anti-CD20 $m$ Abs that are effective not only in flow cytometry but also in Western blot and immunohistochemical analyses. We recently 
Table I. Results of TrMab-6 and EPR20043 immunostaining in 61 breast cancers.

HER 2/

Case Age Sex Pathological diagnosis $\quad$ Differentiation $\quad$ TNM $\quad$ ER PR H$_{2}$ Mab-77 TrMab-6 EPR20043

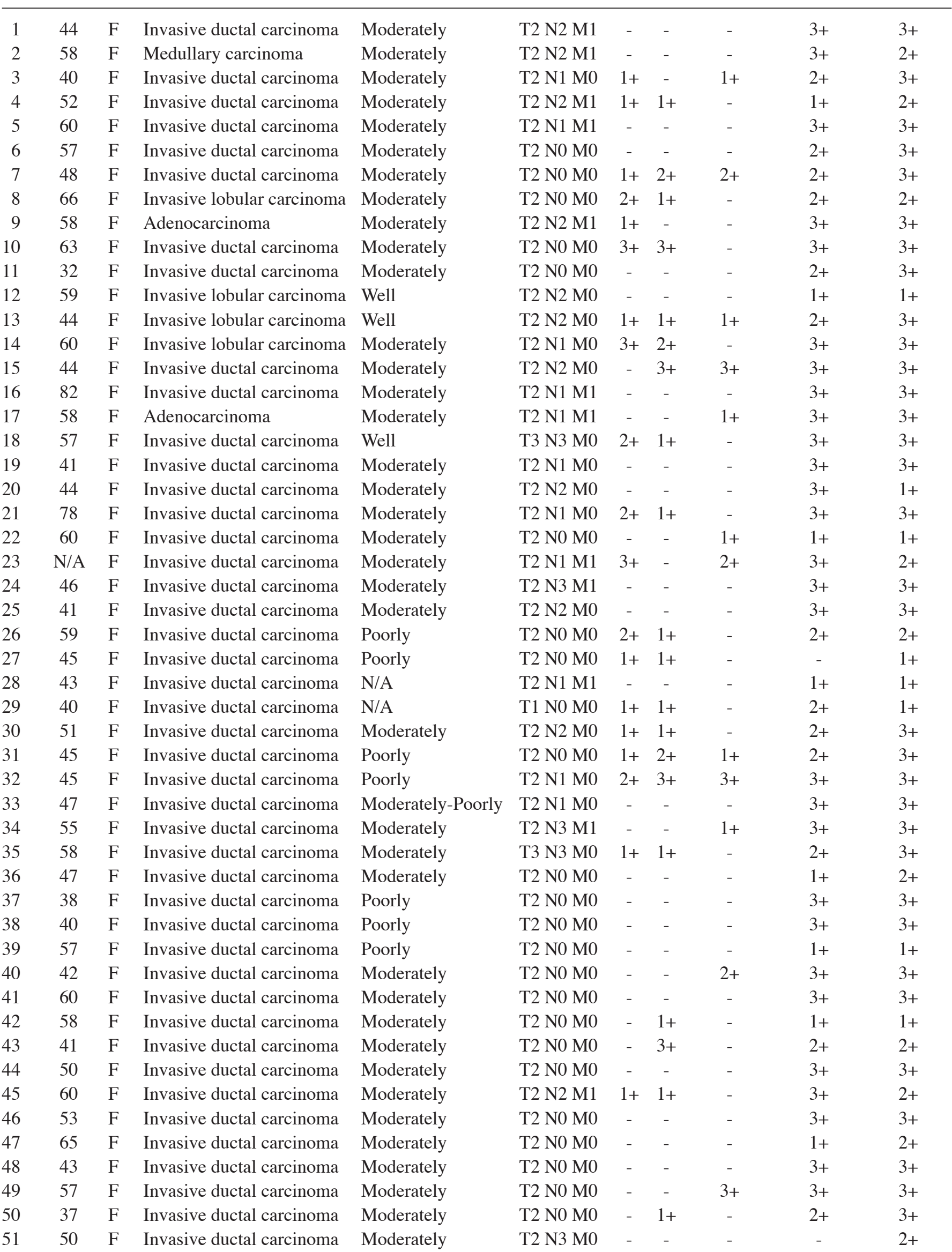


Table I. Continued.

\begin{tabular}{|c|c|c|c|c|c|c|c|c|c|c|}
\hline Case & Age & Sex & Pathological diagnosis & Differentiation & TNM & ER & PR & $\begin{array}{c}\text { HER 2/ } \\
\mathrm{H}_{2} \text { Mab-77 }\end{array}$ & TrMab-6 & EPR20043 \\
\hline 52 & 48 & $\mathrm{~F}$ & Invasive ductal carcinoma & Poorly & T2 N1 M0 & - & - & - & $1+$ & $1+$ \\
\hline 53 & 50 & $\mathrm{~F}$ & Invasive ductal carcinoma & Moderately & T2 N0 M0 & - & - & - & $3+$ & $3+$ \\
\hline 54 & 53 & $\mathrm{~F}$ & Invasive ductal carcinoma & Moderately & T2 N0 M0 & - & - & - & $3+$ & $3+$ \\
\hline 55 & 49 & $\mathrm{~F}$ & Invasive ductal carcinoma & Moderately & T2 N0 M0 & - & - & - & $2+$ & $2+$ \\
\hline 56 & 65 & $\mathrm{~F}$ & Invasive ductal carcinoma & Moderately & T2 N1 M0 & $1+$ & $1+$ & - & $2+$ & $3+$ \\
\hline 57 & 43 & $\mathrm{~F}$ & Invasive ductal carcinoma & Moderately & T2 N0 M0 & - & - & - & - & - \\
\hline 58 & 58 & $\mathrm{~F}$ & Invasive ductal carcinoma & Moderately & T2 N0 M0 & - & - & - & - & $1+$ \\
\hline 59 & 48 & $\mathrm{~F}$ & Invasive ductal carcinoma & Moderately & T2 N0 M0 & - & - & - & $2+$ & $3+$ \\
\hline 60 & N/A & $\mathrm{F}$ & Invasive ductal carcinoma & Moderately & N/A & - & - & - & $2+$ & $2+$ \\
\hline 61 & N/A & $\mathrm{F}$ & Invasive ductal carcinoma & Moderately-Poorly & N/A & $1+$ & $1+$ & - & $2+$ & $3+$ \\
\hline
\end{tabular}

N/A, not available; TNM, tumor node metastasis; F, female; ER, estrogen receptor; PR, progesterone receptor.

Table II. Results of TrMab-6 immunostaining in 61 breast cancers .

TrMab-6

\begin{tabular}{lcccccc}
\cline { 3 - 5 } Pathological diagnosis & No. of cases & $3+$ & $2+$ & $1+$ & - & No. of positive cases (\%) \\
\hline Invasive ductal carcinoma & 54 & 26 & 16 & 8 & 4 & $50 / 54(92.6)$ \\
Invasive lobular carcinoma & 4 & 1 & 2 & 1 & 0 & $4 / 4(100)$ \\
Adenocarcinoma & 2 & 2 & 0 & 0 & 0 & $2 / 2(100)$ \\
Medullary carcinoma & 1 & 1 & 0 & 0 & 0 & $1 / 1(100)$ \\
Total & 61 & $30 / 61(49.2 \%)$ & $18 / 61(29.5 \%)$ & $9 / 61(14.8 \%)$ & $4 / 61(6.6 \%)$ & $57 / 61(93.4 \%)$ \\
\hline
\end{tabular}

Table III. Results of TrMab-6 immunostaining in 31 triple negative breast cancers.

TrMab-6

\begin{tabular}{lcccccc}
\cline { 3 - 5 } Breast cancer subtype & No. of cases & $3+(\%)$ & $2+(\%)$ & $1+(\%)$ & $-(\%)$ & No. of positive cases \\
\hline Triple negative breast cancer & 31 & $17(54.8)$ & $5(16.1)$ & $6(19.4)$ & $3(9.7)$ & $28 / 31(90.3 \%)$ \\
\hline
\end{tabular}

developed clone $\mathrm{C}_{20} \mathrm{Mab}-11$, which can detect $\mathrm{CD} 20$ associated with B-cell lymphoma by flow cytometry, Western blot, and immunohistochemical analyses (24).

Likewise, we herein aimed to establish one or more multipurpose anti-TROP 2 mAbs because the applications of commercially available anti-TROP $2 \mathrm{mAbs}$ were somewhat limited (Table SI). Using the CBIS method, we successfully developed a sensitive and specific novel anti-TROP2 $\mathrm{mAb}$ (clone TrMab-6) that can be used in every application, including flow cytometry (Fig. 3), Western blot (Fig. 4), and immunohistochemical analyses (Fig. 5). Another anti-TROP2 $\mathrm{mAb}$ (clone EPR20043 from Abcam) is more sensitive than TrMab-6 in immunohistochemical analysis (Table I), but not useful in flow cytometry (Fig. S2). EPR20043 might react with intracellular region of TROP 2 although the immunogen was not clearly shown in its application sheet. Clone SP293 (Abcam) was also shown to be useful for flow cytometry, Western blot, and immunohistochemical analyses in its application sheet; however, the intracellular region of TROP2 was also used as immunogen (Table SI).

We conclude that TrMab-6 is more advantageous than the other anti-TROP2 mAbs, such as EPR20043 and SP293 because TrMab-6 is useful to detect TROP2 in all applications, such as flow cytometry in non-fixed condition, Western blot, and immunohistochemical analysis. However, in this study, TrMab-6 was shown to be useful for only in vitro experiments. In the future, we will determine whether TrMab- 6 would be suitable for use as targeted molecular therapy against breast cancers. The subclasses of mouse $\operatorname{IgG}, \operatorname{IgG}_{2 \mathrm{a}}$ and $\mathrm{IgG}_{2 \mathrm{~b}}$, both induce antibody-dependent cellular cytotoxicity (ADCC) or 
complement-dependent cytotoxicity (CDC) $(39,40)$. TrMab-6 was determined to be of the mouse $\mathrm{IgG}_{2 \mathrm{~b}}$ subclass (Fig. S1). Although we performed ADCC reporter assay using TrMab-6, ADCC activity was not observed unexpectedly (data not shown). To promote antibody therapy using TrMab-6, we will utilize antibody-drug conjugates (ADCs), radioimmunotherapy (RIT), photoimmunotherapy (PIT), or chimeric antigen receptor T-cell (CAR-T) therapy. About ADCs targeting TROP2, conjugation of the irinotecan metabolite, SN-38, to a humanized anti-TROP2 antibody (sacituzumab govitecan) promotes broad and potent antitumor effects in human cancer xenografts and in patients with advanced triple-negative breast, non-small-cell and small-cell lung, and urothelial cancers (14). About RIT, van Rij et al (41) previously reported that TROP2-expressing prostate cancer can be targeted efficiently with TF12 [anti-TROP2 $\mathrm{x}$ anti-HSG (histamine-succinyl-glycine)] and ${ }^{177} \mathrm{Lu}$-labeled diHSG-peptide (IMP288). Furthermore, Nishimura et al (42) selected TROP2 as a molecular target for PIT, and utilized a newly developed humanized anti-TROP $2 \mathrm{mAb}$ conjugated to the photosensitizer IR700 (TROP2-IR700) for the treatment of pancreatic carcinoma and cholangiocarcinoma. Growth of tumor xenografts was significantly inhibited in response to TROP2-targeted PIT relative to controls, suggesting that TROP2-targeted PIT is also an important means for improving treatment for TROP2-expressing cancers. About CAR-T, Zhao et al (43) reported that novel bi-specific TROP2/PD-L1 CAR-T cells could target TROP2/PD-L1 and checkpoint blockade, resulting in cytotoxicity for gastric cancer cells. These results also suggested that CAR-T cell therapy featuring TROP2 can be developed to target TROP2-expressing cancers. These various modalities will allow us to explore TrMab-6-mediated antitumor activities in the mouse xenograft model of breast cancer.

\section{Acknowledgements}

The authors would like to thank Mr. Takuro Nakamura, Ms. Miyuki Yanaka, Ms. Saori Handa, Ms. Saki Okamoto, and Mr. Yu Komatsu (Department of Antibody Drug Development, Tohoku University Graduate School of Medicine) for technical assistance in the in vitro experiments.

\section{Funding}

The present study was supported in part by Japan Agency for Medical Research and Development (AMED; grant nos. JP20am0401013, JP20am0101078 and JP20ae0101028).

\section{Availability of data and materials}

The datasets used and/or analyzed during the current study are available from the corresponding author on reasonable request.

\section{Authors' contributions}

YS performed experiments, and analyzed experimental data. YS and YK wrote the manuscript. MKK and YK designed the current study. All authors read and approved the final manuscript.

\section{Ethics approval and consent to participate}

Animal studies were approved by The Animal Care and Use Committee of Tohoku University (Permit no. 2019NiA-001).

\section{Patient consent for publication}

Not applicable.

\section{Competing interests}

The authors declare that they have no competing interests.

\section{References}

1. Bray F, Ferlay J, Soerjomataram I, Siegel RL, Torre LA and Jemal A: Global cancer statistics 2018: GLOBOCAN estimates of incidence and mortality worldwide for 36 cancers in 185 countries. CA Cancer J Clin 68: 394-424, 2018.

2. Bardia A, Mayer IA, Vahdat LT, Tolaney SM, Isakoff SJ, Diamond JR, O'Shaughnessy J, Moroose RL, Santin AD, Abramson VG, et al: Sacituzumab govitecan-hziy in refractory metastatic triple-negative breast cancer. N Engl J Med 380: 741-751, 2019.

3. Anders CK, Zagar TM and Carey LA: The management of early-stage and metastatic triple-negative breast cancer: A review. Hematol Oncol Clin North Am 27: 737-749, viii, 2013.

4. Trivers KF, Lund MJ, Porter PL, Liff JM, Flagg EW, Coates RJ and Eley JW: The epidemiology of triple-negative breast cancer, including race. Cancer Causes Control 20: 1071-1082, 2009.

5. Plasilova ML, Hayse B, Killelea BK, Horowitz NR, Chagpar AB and Lannin DR: Features of triple-negative breast cancer: Analysis of 38,813 cases from the national cancer database. Medicine (Baltimore) 95: e4614, 2016.

6. Kohler BA, Sherman RL, Howlader N, Jemal A, Ryerson AB, Henry KA, Boscoe FP, Cronin KA, Lake A, Noone AM, et al: Annual report to the nation on the status of cancer, 1975-2011, featuring incidence of breast cancer subtypes by race/ethnicity, poverty, and state. J Natl Cancer Inst 107: djv048, 2015.

7. DeSantis CE, Fedewa SA, Goding Sauer A, Kramer JL, Smith RA and Jemal A: Breast cancer statistics, 2015: Convergence of incidence rates between black and white women. CA Cancer J Clin 66: 31-42, 2016.

8. Fornaro M, Dell'Arciprete R, Stella M, Bucci C, Nutini M, Capri MG and Alberti S: Cloning of the gene encoding Trop-2, a cell-surface glycoprotein expressed by human carcinomas. Int J Cancer 62: 610-618, 1995.

9. Alberti S, Miotti S, Stella M, Klein CE, Fornaro M, Menard S and Colnaghi MI: Biochemical characterization of Trop-2, a cell surface molecule expressed by human carcinomas: Formal proof that the monoclonal antibodies T16 and MOv-16 recognize Trop-2. Hybridoma 11: 539-545, 1992.

10. Lipinski M, Parks DR, Rouse RV and Herzenberg LA: Human trophoblast cell-surface antigens defined by monoclonal antibodies. Proc Natl Acad Sci USA 78: 5147-5150, 1981.

11. Schon MP and Orfanos CE: Transformation of human keratinocytes is characterized by quantitative and qualitative alterations of the T-16 antigen (Trop-2, MOv-16). Int J Cancer 60: 88-92, 1995.

12. Zaman S, Jadid H, Denson AC and Gray JE: Targeting Trop-2 in solid tumors: Future prospects. Onco Targets Ther 12: 1781-1790, 2019.

13. Guan H, Guo Z, Liang W, Li H, Wei G, Xu L, Xiao H and Li Y: Trop2 enhances invasion of thyroid cancer by inducing MMP2 through ERK and JNK pathways. BMC Cancer 17: 486, 2017.

14. Goldenberg DM, Stein R and Sharkey RM: The emergence of trophoblast cell-surface antigen 2 (TROP-2) as a novel cancer target. Oncotarget 9: 28989-29006, 2018.

15. Goldenberg DM, Cardillo TM, Govindan SV, Rossi EA and Sharkey RM: Trop-2 is a novel target for solid cancer therapy with sacituzumab govitecan (IMMU-132), an antibody-drug conjugate (ADC). Oncotarget 6: 22496-22512, 2015.

16. Goldstein AS, Huang J, Guo C, Garraway IP and Witte ON: Identification of a cell of origin for human prostate cancer. Science 329: 568-571, 2010. 
17. Goldstein AS, Lawson DA, Cheng D, Sun W, Garraway IP and Witte ON: Trop2 identifies a subpopulation of murine and human prostate basal cells with stem cell characteristics. Proc Natl Acad Sci USA 105: 20882-20887, 2008.

18. King GT, Eaton KD, Beagle BR, Zopf CJ, Wong GY, Krupka HI, Hua SY, Messersmith WA and El-Khoueiry AB: A phase 1, dose-escalation study of PF-06664178, an anti-Trop-2/Aur0101 antibody-drug conjugate in patients with advanced or metastatic solid tumors. Invest New Drugs 36: 836-847, 2018.

19. Cardillo TM, Govindan SV, Sharkey RM, Trisal P, Arrojo R, Liu D, Rossi EA, Chang CH and Goldenberg DM: Sacituzumab govitecan (IMMU-132), an anti-trop-2/SN-38 antibody-drug conjugate: Characterization and efficacy in pancreatic, gastric, and other cancers. Bioconjug Chem 26: 919-931, 2015.

20. Cardillo TM, Govindan SV, Sharkey RM, Trisal $P$ and Goldenberg DM: Humanized anti-Trop-2 IgG-SN-38 conjugate for effective treatment of diverse epithelial cancers: Preclinical studies in human cancer xenograft models and monkeys. Clin Cancer Res 17: 3157-3169, 2011.

21. Okajima D, Yasuda S, Yokouchi Y, Fujitani T, Sakurai K and Yamaguchi J: Preclinical efficacy studies of DS-1062a, a novel TROP2-targeting antibody-drug conjugate with a novel DNA topoisomerase I inhibitor DXd. J Clinical Oncol 36 (Suppl 15): e24206, 2018.

22. Itai S, Fujii Y, Nakamura T, Chang YW, Yanaka M, Saidoh N, Handa S, Suzuki H, Harada H, Yamada S, et al: Establishment of CMab-43, a sensitive and specific anti-CD133 monoclonal antibody, for immunohistochemistry. Monoclon Antib Immunodiagn Immunother 36: 231-235, 2017.

23. Yamada S, Kaneko MK, Sayama Y, Asano T, Sano M, Yanaka M, Nakamura T, Okamoto S, Handa S, Komatsu Y, et al: Development of novel mouse monoclonal antibodies against human CD19. Monoclon Antib Immunodiagn Immunother 39: 45-50, 2020

24. Furusawa Y, Kaneko MK and Kato Y: Establishment of C20Mab-11, a novel anti-CD20 monoclonal antibody, for the detection of B cells. Oncol Lett 20: 1961-1967, 2020.

25. Yamada S, Itai S, Nakamura T, Yanaka M, Kaneko MK and Kato Y: Detection of high CD44 expression in oral cancers using the novel monoclonal antibody, C(44)Mab-5. Biochem Biophys Rep 14: 64-68, 2018.

26. Yamada S, Itai S, Nakamura T, Yanaka M, Chang YW, Suzuki H, Kaneko MK and Kato Y: Monoclonal antibody L(1)Mab-13 detected human PD-L1 in lung cancers. Monoclon Antib Immunodiagn Immunother 37: 110-115, 2018.

27. Fujii Y, Kaneko M, Neyazaki M, Nogi T, Kato Y and Takagi J: PA tag: A versatile protein tagging system using a super high affinity antibody against a dodecapeptide derived from human podoplanin. Protein Expr Purif 95: 240-247, 2014.

28. Fujii Y, Kaneko MK, Ogasawara S, Yamada S, Yanaka M, Nakamura T, Saidoh N, Yoshida K, Honma R and Kato Y: Development of RAP Tag, a novel tagging system for protein detection and purification. Monoclon Antib Immunodiagn Immunother 36: 68-71, 2017.

29. Fujii Y, Kaneko MK and Kato Y: MAP Tag: A novel tagging system for protein purification and detection. Monoclon Antib Immunodiagn Immunother 35: 293-299, 2016.

30. Kohler G and Milstein C: Continuous cultures of fused cells secreting antibody of predefined specificity. Nature 256: 495-497, 1975.

31. Kato Y, Kaneko MK, Kuno A, Uchiyama N, Amano K, Chiba Y, Hasegawa Y, Hirabayashi J, Narimatsu H, Mishima K and Osawa M: Inhibition of tumor cell-induced platelet aggregation using a novel anti-podoplanin antibody reacting with its platelet-aggregation-stimulating domain. Biochem Biophys Res Commun 349: 1301-1307, 2006.
32. Itai S, Fujii Y, Kaneko MK, Yamada S, Nakamura T, Yanaka M, Saidoh N, Chang YW, Handa S, Takahashi M, et al: H2Mab-77 is a sensitive and specific Anti-HER2 monoclonal antibody against breast cancer. Monoclon Antib Immunodiagn Immunother 36: 143-148, 2017.

33. Furusawa Y, Yamada S, Itai S, Nakamura T, Yanaka M, Sano M, Harada H, Fukui M, Kaneko MK and Kato Y: PMab-219: A monoclonal antibody for the immunohistochemical analysis of horse podoplanin. Biochem Biophys Rep 18: 100616, 2019.

34. Furusawa Y, Kaneko MK, Nakamura T, Itai S, Fukui M, Harada H, Yamada S and Kato Y: Establishment of a monoclonal antibody PMab-231 for tiger podoplanin. Monoclon Antib Immunodiagn Immunother 38: 89-95, 2019.

35. Furusawa Y, Takei J, Sayama Y, Yamada S, Kaneko MK and Kato Y: Development of an anti-bear podoplanin monoclonal antibody PMab-247 for immunohistochemical analysis. Biochem Biophys Rep 18: 100644, 2019.

36. Furusawa Y, Yamada S, Itai S, Nakamura T, Takei J, Sano M, Harada H, Fukui M, Kaneko MK and Kato Y: Establishment of a monoclonal antibody PMab-233 for immunohistochemical analysis against Tasmanian devil podoplanin. Biochem Biophys Rep 18: 100631, 2019.

37. Polyak MJ, Li H, Shariat N and Deans JP: CD20 homo-oligomers physically associate with the B cell antigen receptor. Dissociation upon receptor engagement and recruitment of phosphoproteins and calmodulin-binding proteins. J Biol Chem 283: 18545-18552, 2008.

38. Li H, Ayer LM, Lytton J and Deans JP: Store-operated cation entry mediated by CD20 in membrane rafts. J Biol Chem 278: 42427-42434, 2003.

39. Kaneko MK, Nakamura T, Honma R, Ogasawara S, Fujii Y, Abe S, Takagi M, Harada H, Suzuki H, Nishioka Y and Kato Y: Development and characterization of anti-glycopeptide monoclonal antibodies against human podoplanin, using glycan-deficient cell lines generated by CRISPR/Cas9 and TALEN. Cancer Med 6: 382-396, 2017.

40. Ogasawara S, Kaneko MK and Kato Y: LpMab-19 recognizes sialylated O-Glycan on Thr76 of human podoplanin. Monoclon Antib Immunodiagn Immunother 35: 245-253, 2016.

41. van Rij CM, Frielink C, Goldenberg DM, Sharkey RM, Lütje S, McBride WJ, Oyen WJG and Boerman OC: Pretargeted radioimmunotherapy of prostate cancer with an anti-TROP-2xAnti-HSG bispecific antibody and a (177)Lu-labeled peptide. Cancer Biother Radiopharm 29: 323-329, 2014.

42. Nishimura T, Mitsunaga M, Sawada R, Saruta M, Kobayashi H, Matsumoto N, Kanke T, Yanai $\mathrm{H}$ and Nakamura $\mathrm{K}$ : Photoimmunotherapy targeting biliary-pancreatic cancer with humanized anti-TROP2 antibody. Cancer Med 8: 7781-7792, 2019.

43. Zhao W, Jia L, Zhang M, Huang X, Qian P, Tang Q, Zhu J and Feng Z: The killing effect of novel bi-specific Trop2/PD-L1 CAR-T cell targeted gastric cancer. Am J Cancer Res 9: 1846-1856, 2019

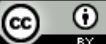

This work is licensed under a Creative Commons Attribution 4.0 International (CC BY 4.0) License. 